\title{
A TEORIA FUNDAMENTADA NOS DADOS NA PESQUISA EM ADMINISTRAÇÃO: EVIDÊNCIAS E REFLEXÕES
}

\section{The Grounded Theory in Management Research: Evidences and Reflections}

\section{Angélica Pott de Medeiros}

Universidade Federal de Santa Maria (UFSM).

email:apm_angelica@yahoo.com.br

\section{José Luís Guedes dos Santos}

Universidade Federal de Santa Catarina (UFSC).

email:joseenfermagem@gmail.com

\section{Rolf Hermann Erdmann}

Universidade Federal de Santa Catarina (UFSC).

email: rolf.erdmann@ufsc.br

\section{RESUMO}

O presente estudo teve como objetivo analisar as publicações brasileiras na área de administração que utilizaram a Teoria Fundamentada nos Dados (TFD) como método, buscando fazer um panorama acerca da utilização deste no país e identificando as suas principais características. Para isso, foi realizada uma revisão integrativa de literatura, a partir da consulta da base de dados de anais dos congressos da ANPAD e a plataforma de dados Spell. A análise baseou-se na caracterização da amostra, bem como quatorze elementos da TFD. Os resultados demonstram uma concentração de autores doutores oriundos da região sul e sudeste do país. Além da redução da utilização do método nos últimos anos. Ainda, observou-se que muitos estudos não se aprofundaram no detalhamento da utilização do método. Constata-se que a TFD consiste em um método complexo, e que demanda de muita dedicação e tempo do pesquisador, devido a imersão aos dados. Diante disso, fica evidente a necessidade de uma maior discussão acerca do método em eventos científicos, além da introdução deste em programas de pós-graduação em administração no país.

Palavras-chave: Teoria Fundamentada nos Dados; Administração; Revisão Integrativa de Literatura.

\section{ABSTRACT}

The present study aimed to analyze the Brazilian publications in the area of management that used Grounded Theory (GT) as a method, seeking to provide an overview of its use in the country and identifying its main characteristics. For that, an integrative literature review was carried out, based on the consultation of the ANPAD congress annals database and the Spell data platform. The analysis was based on the characterization of the sample, as well as fourteen elements of the TFD. The results demonstrate a concentration of doctoral authors from the south and southeast of the country. In addition to the reduction in the use of the method in recent years. Still, it was observed that many studies did not go into the details of the use of the method. It appears that PDT consists of a complex method, and that it demands a lot of dedication and time from the researcher, due to the immersion in the data. Given this, it is evident the need for a greater discussion about the method in scientific events, in addition to the its introduction in graduate programs in management in the country.

Keywords: Grounded Theory; Management; Integrative literature review. 


\section{INTRODUÇÃO}

Diferentes tradições de pesquisa classificam-se como qualitativas, ambas compartilham o pressuposto básico de que a investigação de fenômenos humanos possui singularidades, ou seja, através dela é possível analisar as pessoas em suas interações sociais, bem como os significados que são atribuídos nessas interações. Opondo-se aos pressupostos quantitativos, os quais consideram a quantificação como forma de generalização e de validade, os quais são oriundos das ciências naturais. Apesar disso, muitas orientações filosóficas e epistemológicas direcionaram-se para a pesquisa qualitativa, a partir de diferentes métodos de pesquisa, como a observação participante, entrevista, história de vida, análise de discurso, etnografia, teoria fundamentada nos dados, pesquisa-ação, entre outros (CHIZZOTTI, 2003).

Glaser e Strauss desafiaram o paradigma positivista de sua época, que defendia que a pesquisa qualitativa era, sobretudo, tendenciosa e assistemática. Os autores provaram a partir de seus trabalhos que a pesquisa qualitativa pode ir além da mera descrição dos resultados, e que é possível conceber explicações teóricas acerca do comportamento humano. Diante disso, a partir da abordagem sistemática da teoria fundamentada nos dados provou-se que os resultados da pesquisa qualitativa condizem com o que é observado na realidade (MELLO; CUNHA, 2010).

A Grounded Theory ou Teoria Fundamentada nos Dados (TFD) consiste em um método indutivo-dedutivo. Seu foco central é a busca pela compreensão de experiências e interações dos indivíduos inseridos em um determinado contexto social. Foi desenvolvida em meados dos anos de 1960 por Barney Glaser e Anselm Strauss. De um lado, Glaser era originário da Universidade de Columbia, com interesse em métodos empíricos e na teoria sociológica. Por outro lado, Strauss tinha formação acadêmica pela Universidade de Chicago, a qual apresentava tradição qualitativa e abordagens críticas (SANTOS et al., 2016).

O método foi desenvolvido na junção das escolas de pensamento de Glaser e Strauss, a partir de um estudo sobre o relacionamento entre médicos e pacientes terminais. A medida em que o estudo avançava, os autores foram desenvolvendo estratégias metodológicas sistemáticas que podiam ser utilizadas por outros pesquisadores, o que deu origem ao livro "The Discovery of Grounded Theory” de 1967. Esse livro descreve as estratégias metodológicas e o desenvolvimento de teorias a partir de dados. Assim, a denominada Grounded Theory se consolidava como um processo contínuo e sistemático de coleta e análise de dados para a geração e a verificação dos resultados. O produto desse método é um modelo descritivo de um fenômeno, ou da influência desse fenômeno em um processo social (MELLO; CUNHA, 2010).

Além das especificidades que o método apresenta, os autores apresentam divergências no que tange as estratégias para a implementação da TFD. Haja vista as rupturas entre os autores do método, a TFD apresenta-se atualmente em três vertentes principais, a Clássica que segue os pressupostos de Glaser, a Straussiana, fundada por Strauss a partir de sua ruptura com Glaser, e a vertente Construtivista, esquematizado por Kathy Charmaz a partir dos anos 2000.

Conforme argumenta Santos (2018), tem-se observado novos movimentos acerca da TFD, como a análise situacional de Adele Clarke, como uma derivação pós-moderna do método, vertente que tem sido utilizada em pesquisas sobre gênero e sexualidade. E a análise dimensional, oriunda dos estudos de Leonard Schatzman e Strauss, a qual, pauta-se na dificuldade de gerar categorias a partir dos dados, contando com a identificação prévia do pesquisador das dimensões características do seu campo de estudo.

Evidencia-se também, que a literatura acerca do método é extensa, sobretudo internacional. No Brasil o uso desse método se dá em grande medida nas áreas de sociologia, psicologia e enfermagem (GOMES et al., 2015). Bianchi e Ikeda (2008) argumentam que na área da administração a TFD começou a ser utilizada somente a partir dos anos 1990, porém, ainda se verificam poucos estudos que utilizam o método.

Diante da extensa possibilidade da utilização da TFD na administração, especialmente dada a possibilidade de análise das interrelações entre os sujeitos, e a compreensão de fenômenos organizacionais (BANDEIRA-DE-MELLO; CUNHA, 2006), combinado com o reduzido volume de pesquisas brasileiras que baseiam-se na TFD, emerge a questão central dessa pesquisa: Quais são as características principais das 
publicações brasileiras na área de administração que utilizaram a TFD como método? Para responder essa indagação, o objetivo proposto é analisar as publicações brasileiras na área de administração que fizeram o uso da TFD, com base numa revisão integrativa de literatura.

A partir disso, busca-se constituir um panorama acerca da utilização do método no país, identificando suas principais características e elementos. Contribuindo com a identificação das lacunas e dos requisitos essenciais do método, visando auxiliar nas pesquisas sobre o tema. Ressalta-se que estudos semelhantes a esse já foram realizados em outras áreas de concentração como a enfermagem (GOMES et al., 2015; SANTOS et al., 2016; BARRETO et al., 2018), e na administração, porém em grande parte são trabalhos teóricos que exploram sobre usos e aplicações do método (PREDEBON; DE SOUZA, 2006; BIACHI; IKEDA, 2008; LOURENÇO et al., 2008; PINTO; SANTOS, 2008; PETRINI; POZZEBON, 2009; IKEDA; BIACHI, 2009; PREDEBON et al., 2011; HALAWEH, 2012; PINTO; SANTOS, 2012; MIGUEL; POPADIUK, 2014).

Além destes, destaca-se estudos de levantamento bibliométrico como o estudo de Pinto, de Freitas e Mendes (2016) que focaram sua análise no período de 1997 e 2014. De Castro e Machado (2017) cuja análise incluiu artigos de 2000 a 2014. E, Berto e Erdmann (2017) que analisaram o uso da TFD na geração de teoria substantiva nos estudos da administração publicados até 2014. Apesar disso, destaca-se que o presente estudo apresenta uma análise mais ampla, e busca comparar os pressupostos metodológicos da TFD com o que realmente é observado na literatura. Ademais, não se delimita um período inicial de análise perfazendo-se até setembro de 2018, data da realização do levantamento. Também, leva-se em consideração todas as revistas que fazem parte do banco de dados, não fazendo distinção pela classificação.

Afora esta introdução, o texto está estruturado em outras quatro seções. Na sequência delineia-se sobre as principais características da teoria fundamentada nos dados, bem como os estudos já desenvolvidos sobre o método na área de administração. Na terceira seção a abordagem metodológica é detalhada, a qual consiste em uma revisão integrativa de literatura. $\mathrm{Na}$ seção seguinte, são apresentados os resultados e discussões do estudo, e na quarta seção são delineadas as principais conclusões do estudo.

\section{TEORIA FUNDAMENTADA NOS DADOS: PRINCIPAIS CARACTERÍSTICAS}

Desde o lançamento de "The Discovery of Grounded Theory" em 1967, obra na qual a teoria fundamentada nos dados foi introduzida, diferentes vertentes foram apresentadas na literatura com o passar dos anos. Muitas das divergências centravam-se na relação entre indução e dedução, análise de dados e a formação da teoria (HEATH; COWLEY, 2004). Contudo, ambos os autores defendem que a metodologia busca a interpretação de significados, eventos, experiências e realidades, para a profunda compreensão dos fenômenos sociais (HARRIS, 2015).

Kenny e Fourie (2015) destacam que as três principais vertentes da TFD: Clássica, Straussiana e Construtivista, não são homogêneas ou intercambiáveis. Conforme observa-se na Figura 1, suas diferenças centram-se em três aspectos principais: a) procedimentos de codificação; b) posições filosóficas; e c) utilização da literatura.

Além de posições filosóficas distintas, Gomes et al. (2015) argumentam que a análise de dados, e inerentemente a codificação dos dados, apresentam divergências nas vertentes da TFD, apesar disso consiste num aspecto crucial do método. Em relação ao uso da literatura, Glaser e Strauss (1967) defendem que no início do desenvolvimento do estudo, o pesquisador só deve ter claro questões referentes a coleta inicial dos dados, no lugar da predeterminação do procedimento como um todo, haja vista a perspectiva de que os dados revelarão a necessidade de novas procedimentos de coleta de dados. Destaca-se que essa necessidade de mais dados surge durante diferentes estágios da pesquisa. Primeiramente, haja vista que os dados passam por um processo de codificação e categorização, é possível identificar as lacunas, indicando a necessidade de novas evidências. Por outro lado, nas demais vertentes observa-se maior flexibilidade em relação à utilização da literatura. 
Figura 1 Princípios unificadores e diferenciadores da TFD

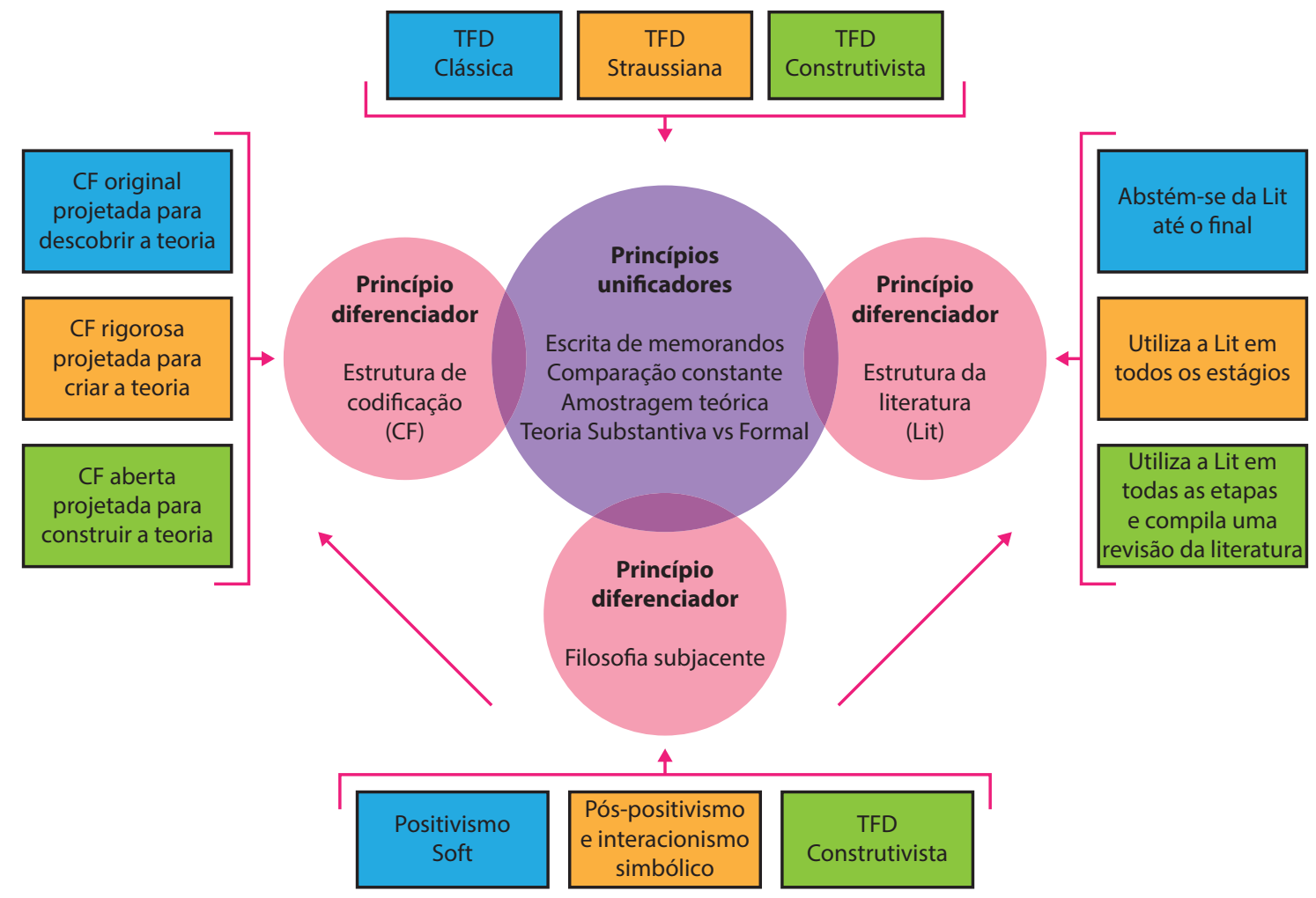

\footnotetext{
Observações

- O círculo roxo engloba os princípios unificadores das três vertentes da TFD.

- Os três círculos rosa indicam as três áreas de contenção onde as três vertentes discordam.

- As caixas azuis significam posições da TFD Clássica.

- As caixas laranja significam posições da TFD Straussiana.

- As caixas verdes significam posições da TFD Construtivista.

- As setas rosa indicam a influência de um preceito contestado em outro.

- CF é um acrônimo para Estrutura de Codificação; Lit é um acrônimo para Literatura.
}

Fonte Adaptado de Kenny e Fourie (2015).

Além dos princípios diferenciadores entre as vertentes, a TFD em suas diferentes tradições, apresenta também similaridades, as quais concentram-se na utilização de memorandos pelo pesquisador, comparação constante, amostragem teórica, e a distinção entre teoria formal e teoria substantiva (KENNY; FOURIE, 2015).

A amostragem teórica parte do pressuposto que com o processo de coleta de dados, codificação e análise simultânea, conceitos inesperados podem surgir, redirecionando o estudo, e por consequência, exigindo novos dados. Ademais, a medida em que a teoria emerge, o pesquisador pode identificar novas lacunas. Assim, a amostra da pesquisa será guiada a partir dessas identificações, ao invés de ser predeterminada no início. Esse fenômeno é conhecido como processo evolutivo da amostragem teórica. Procedimento que se perpetua até a saturação, momento em que nenhum novo dado está surgindo (GLASER; STRAUSS, 1967).

Esses princípios são intrínsecos das três principais vertentes da TFD (Clássica, Straussiana e Contrutivista), pois cada uma delas estabelece que a amostra da pesquisa não pode ser predeterminada, em vez disso, deve-se seguir os pressupostos da amostragem teórica, coordenada pela teoria emergente até o momento de saturação teórica (KENNY; FOURIE, 2015).

De outro modo, o Quadro 1 ilustra também as características centrais da TFD. Evidencia-se que os três aspectos diferenciadores explanados anteriormente influenciam nas demais características, como o processo de identificação do problema de pesquisa, sua condução da investigação e a relação entre o pesquisador e o pesquisado nas diferentes vertentes. 
Quadro 1 Características centrais da TFD

\begin{tabular}{c|l|l|l}
\hline \multicolumn{1}{|c|}{ Clássica } & \multicolumn{1}{c|}{ Straussiana } & \multicolumn{1}{c}{ Construtivista } \\
\hline Paradigma epistemológico & Positivismo & Pós-positivismo & Construtivismo \\
\hline $\begin{array}{c}\text { Identificação do problema de } \\
\text { pesquisa }\end{array}$ & $\begin{array}{l}\text {-Emergente } \\
\text {-Sem a necessidade de aprofun- } \\
\text { damento na revisão de literatura } \\
\text { inicial }\end{array}$ & $\begin{array}{l}\text {-Experiência } \\
\text {-Pragmatismo } \\
\text {-Literatura }\end{array}$ & $\begin{array}{l}\text {-Sensibilização de conceitos } \\
\text {-Específicos de cada disciplina }\end{array}$ \\
\hline $\begin{array}{c}\text { Condução da investigação e } \\
\text { desenvolvimento da teoria }\end{array}$ & $\begin{array}{l}\text { Emergência dos dados através } \\
\text { da indução e da criatividade }\end{array}$ & $\begin{array}{l}\text { Modelo paradigmático de } \\
\text { verificação }\end{array}$ & $\begin{array}{l}\text { Coconstrução e reconstrução de } \\
\text { dados para a teoria }\end{array}$ \\
\hline Relação com os participantes & Independente & Ativa & Coconstrução \\
\hline Análise dos dados/codifica- & $\begin{array}{l}\text {-Aberta } \\
\text {-Axial } \\
\text {-Teórica }\end{array}$ & $\begin{array}{l}\text {-Aberta } \\
\text {-Axial }\end{array}$ & $\begin{array}{l}\text {-Inicial } \\
\text {-Focalizada }\end{array}$ \\
\hline -Seletiva & $\begin{array}{l}\text {-Ajuste } \\
\text { Avaliação da teoria }\end{array}$ & $\begin{array}{l}\text {-Compreensão } \\
\text {-Generalização teórica } \\
\text { - -Relevância } \\
\text {-Modificabilidade }\end{array}$ & $\begin{array}{l}\text {-Congruência e consistência da } \\
\text { teoria em relação ao contexto } \\
\text {-Interpretação reflexiva do } \\
\text { pesquisador }\end{array}$ \\
\hline
\end{tabular}

Fonte Adaptado de Hunter et al. (2011) e Santos et al. (2016).

De maneira particular a TFD concebeu um modo específico de análise de dados, o se trata do método de comparação constante. Os dados coletados são analisados linha por linha, e cada incidente é codificado através de um rótulo. A partir disso, esses códigos são agrupados em categorias que indicam conceitos. Diante do processo em que o pesquisador coleta, codifica, analisa e categoriza os dados, observa-se que há três níveis de comparação constante: a) os códigos são comparados entre si; b) os códigos são comparados com categorias emergentes; e c) as categorias são comparadas entre si. Além disso, no fim da pesquisa, a teoria emergente é comparada com a literatura (KENNY; FOURIE, 2015). Bandeira-de-Mello e Cunha (2006) ilustram o método de comparação constante através da Figura 2.

Figura 2 Método de comparação constante

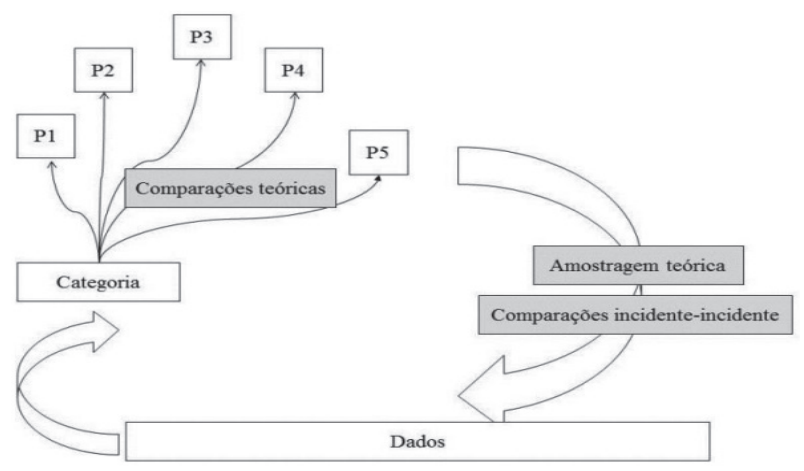

Fonte Bandeira-de-Mello e Cunha (2006, p. 251).
O modelo exposto, evidencia a ênfase na circularidade entre as coletas e análise de dados. As siglas $\mathrm{P} 1$, P2, P3, P4 e P5 referem-se a propriedades provisórias, oriundas das comparações teóricas e da introspecção do pesquisador, as quais devem ser validadas nos dados. O diferencial dessa metodologia consiste na presença de elementos dedutivos e abdutivos. Através da lógica abdutiva os dados coletados no campo de estudo são organizados de forma indutiva. A partir disso são determinadas categorias específicas e relações para inferir no primeiro esboço da teoria substantiva que se pretende gerar. Conforme a Figura 3, a partir desse primeiro esboço retorna-se ao campo para verificar como ele se comporta diante dos novos dados coletados, os quais, possibilitarão, ou não, novas deduções até chegar do modelo teórico final (BANDEIRA-DE-MELLO; CUNHA, 2006).

Figura 3 Indução, dedução e verificação na TFD

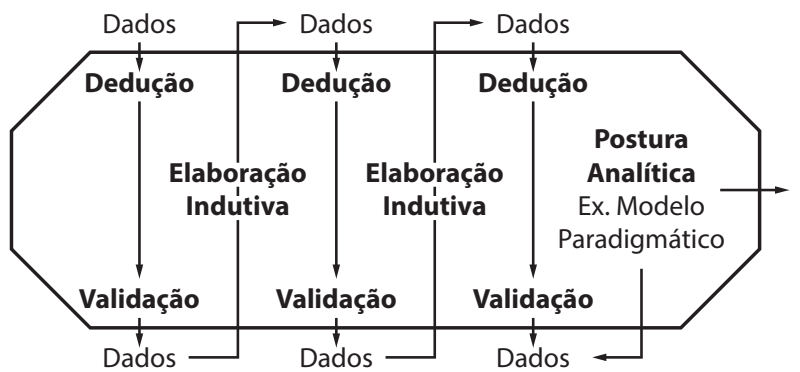

Fonte Adaptado de Strauss e Corbin (1998). 
Além do pioneirismo na comparação constante, a TFD introduziu a técnica do memorando escrito. $\mathrm{Na}$ medida em que os conceitos vão emergindo dos dados e da comparação constante, o pesquisador reflete sobre essas informações construindo memorandos de suas reflexões. E, os diagramas expressam em forma de ilustrações essas reflexões. Esse processo facilita a teorização, por isso, pode-se dizer que a redação de memorandos é intrínseco da TFD, independentemente da sua vertente (GOMES et al., 2015).

Santos et al. (2016) expõem que os diagramas consistem em recursos visuais, permitindo a integração das diferentes etapas da pesquisa, e facilitam as conexões entre as categorias da teoria emergente. Enquanto que os memorandos são anotações que buscam o desenvolvimento dos conceitos. Essas ferramentas podem ser utilizadas de forma manual ou por meio de softwares de análise qualitativa, mas o importante é utilizá-las.

Por fim, destaca-se que as três principais vertentes da TFD distinguem a teoria substantiva da teoria formal. A primeira refere-se ao desenvolvimento de teoria acerca de uma área específica e empírica, ou seja, substantiva. Enquanto a segunda gera teoria de uma área formal e conceitual. Ainda, enquanto a teoria substantiva explica determinado fenômeno em um espaço e tempo distinto, a teoria formal ultrapassa essas dimensões. De forma que para gerar teoria formal a partir da TFD é necessária a realização de comparação constante nos diferentes casos substantivos que se enquadram na área formal determinada (GRASER; STRAUSS, 1967).

\subsection{Estudos preliminares acerca da utilização da TFD em Administração}

Apesar de o surgimento da Teoria Fundamentada nos Dados ser datada dos anos de 1960, no Brasil, a área de administração começou a utilizar o método quase trinta anos depois. Diante da abrangência de possibilidades da utilização da TFD nos estudos organizacionais, diferentes estudos já utilizaram o método, além destes, outros estudos foram desenvolvidos visando analisar as principais características dos estudos desenvolvidos em torno da aplicação da TFD. Sob um panorama internacional, Jacobus, de Souza e Bitencourt (2012) analisaram 45 artigos que utilizaram a TFD e foram publicados em periódicos internacionais da área da administração entre 1995 e 2011. O estudo revela que não há uma TFD homogênea, mas um conceito prototípico que se realiza de formas ligeiramente diferentes. A aplicação abrange temas diversos, como inovação, liderança, tomada de decisão, marketing, entre outros. Observou-se uma preocupação quanto a justificativa da utilização do método e os procedimentos para a triangulação, porém não se percebeu, na maioria dos artigos, igual empenho para detalhar o processo de codificação e análise dos dados. Sob essa perspectiva, evidencia-se que quase a totalidade dos artigos analisados não produziram teoria plena, mas aproximações teóricas.

Em análise ao contexto brasileiro, Mendonça et al. (2013) verificaram a ocorrência da aplicação da TFD nos artigos dos eventos da ANPAD (Associação Nacional de Pós-Graduação e Pesquisa em Administração) e nos periódicos de administração classificados entre A1 e B2 no período de 2001 e 2010. O portfólio contou com 55 artigos. Dentre as revistas científicas destaca-se a Organizações \& Sociedade, com três dos oito artigos publicados em periódicos. Em análise a distribuição das publicações por ano, em periódicos observou-se um número constante de publicações no período entre 2001 e 2010, enquanto que em anais de eventos os anos em destaque são os de $2008 \mathrm{com} \mathrm{dez}$ publicações e de 2010 com 11 publicações. Dentre as instituições de filiação dos autores, destaca-se a Fundação Getúlio Vargas de São Paulo, Universidade de São Paulo e Universidade Federal do Paraná. Por fim, observou-se uma maior ocorrência de artigos que utilizaram a técnica de entrevista para a condução das suas pesquisas, e em relação a análise dos dados, a maioria dos estudos que evidenciaram esse aspecto, descreveu os passos de codificação aberta, axial e seletiva, processos de codificação oriundos da vertente Straussiana.

De maneira similar, os estudos de Ramires e Machado (2015); e De Castro e Machado (2017) realizaram um levantamento de artigos publicados entre 2000 e 2014, conforme a listagem do sistema QUALIS da CAPES, com classificação entre A1 e B1, além de artigos científicos publicados nos Anais dos congressos da ANPAD. Foram analisados 20 artigos da área de Administração. Dentre os principais resultados, a conclusão é de que a grounded theory como método de pesquisa não é aplicada de maneira 
uniforme, embora os autores tenham empregado as etapas e os princípios básicos que mantiveram a essência do método. Percebeu-se que todos os artigos analisados neste estudo não produziram uma teoria propriamente dita, ou seja, uma teoria plena, mas, sim produziram aproximações teóricas. Ademais, aplicação do método contemplou temas diversos, entre os quais se destacam estratégia organizacional, sustentabilidade, marketing, consumo, inovação tecnológica e vantagem competitiva.

Pinto, de Freitas e Mendes (2016) realizaram análise bibliométrica de artigos que citaram a teoria fundamentada em um dos principais veículos para divulgação de pesquisas acadêmicas em administração de empresas no Brasil: os anais do Encontro da ANPAD no período entre 1997 e 2014. De acordo com os autores, os resultados indicam que a teoria fundamentada nos estudos de gestão no Brasil apresenta uma pluralidade de vertentes, além de uso indevido, abordagens e entendimentos superficiais e equivocados. Verifica-se que os artigos analisados não dedicam espaço às discussões epistemológicas, $o$ mesmo refere-se à explicação da metodologia.

Mais recentemente, Berto e Erdmann (2017) levantaram os artigos no Portal de Periódicos CAPES, buscando estudos que utilização a TFD na Administração, no período que compreendeu até 2014 . A amostra consistiu em 43 artigos, e os resultados apontaram que um grande volume de publicações ocorreu nos anos de 2009 e 2013, sendo a ápice no ano de 2012. Os artigos, em sua maioria, abrangem temas como gestão educacional e administração geral. Ademais, observou-se que a maioria da amostra apresentou a utilização da TFD como metodologia única de pesquisa, mas, outros seis artigos empregaram outras metodologias conjuntas. Numa análise mais aprofundada, foi possível verificar que sete artigos da amostra apresentam possibilidades de transformar uma teoria substantiva em uma teoria formal.

Diante dessas evidências, constata-se que os resultados estão limitados a levantamentos realizados em periódicos mais relevantes na área, e em eventos ligados a ANPAD, num período que compreende entre 1995 a 2014, fazendo-se necessário uma análise mais ampla e atualizada da aplicação do método, visando apoiar os pesquisadores da área de administração, o que consiste no objetivo desse estudo.

\section{ASPECTOS METODOLÓGICOS}

Tendo em vista que o objetivo do estudo compreende em analisar as publicações brasileiras na área de administração que utilizaram a TFD como método, realizou-se uma revisão integrativa de literatura, seguindo os pressupostos de Mendes et al. (2008), aplicando-se para a área de administração. Conforme as autoras, este método possibilita a síntese de múltiplos estudos publicados, e gera conclusões gerais acerca de uma área específica. Ademais, destaca-se que o revisor avalia criticamente os métodos utilizados no desenvolvimento dos estudos levantados, acarretando na redução do número de estudos incluídos na análise final, que compreende numa análise sistemática.

Figura 4 Etapas da análise integrativa

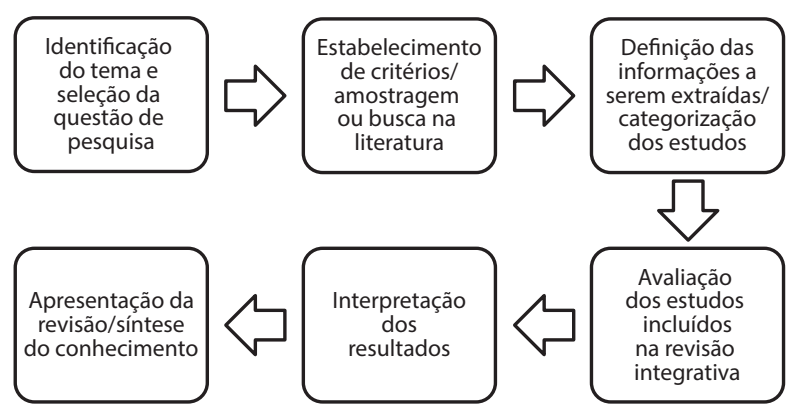

Fonte Mendes et al. (2008), adaptação própria.

Conforme a Figura 4, foram seguidas as seis etapas do método. Inicialmente realizou-se a identificação do tema e a seleção da questão de pesquisa. Uma vez definida a questão de pesquisa foi possível definir os critérios de inclusão ou exclusão dos artigos, bem como a amostragem e a busca na literatura. Desse modo, procedeu-se à escolha das fontes de dados e bases para a realização da pesquisa. Tendo em vista a especificidade da busca na área de administração, optou-se pela base de dados de anais dos congressos da Associação Nacional de Pós-Graduação e Pesquisa em Administração (ANPAD), assim como a plataforma de dados Spell (Scientific Periodicals Electronic Library), desenvolvida pela ANPAD, que disponibiliza gratuitamente a produção científica das áreas de Administração Pública e de Empresas, Contabilidade e Turismo.

Para a localização dos artigos, realizou-se uma busca com as palavras-chave "grounded theory", "teoria fundamentada" e "teoria substantiva". Diante 
disso, na plataforma dos eventos da ANPAD foram localizados 30 artigos, já na base de dados Spell localizou-se 33 artigos. A partir disso, seguiu-se para a seleção conforme os critérios de inclusão: artigos com acesso ao texto completo e em qualquer idioma. Destaca-se que não foi determinado um período, para que a evolução ao longo do tempo pudesse ser observada. Os dados foram coletados em setembro de 2018, assim o intervalo temporal se limita a agosto de 2018. Ademais, foram eliminados os artigos que não compreendiam em relatos empíricos da utilização da Teoria Fundamentada em Dados, e que apenas apresentavam o método teoricamente. A partir desse filtro, restaram 28 artigos, destes 3 foram excluídos pois apresentavam duplicidade, ou seja, foram apresentados em eventos e posteriormente publicados em revistas, optou-se pela versão publicada em periódico.

A etapa seguinte compreendeu na definição das informações a serem extraídas dos estudos selecionados e a categorização dos estudos, baseando-se na revisão bibliográfica acerca do método. Diante disso, buscou-se determinar dados de identificação dos artigos, bem como itens conforme as questões metodológicas da TFD. Dentre os quais destacam-se: vertente utilizada, presença de referencial teórico, coleta de dados, amostragem teórica, saturação teórica dos dados, análise dos dados, critérios de codificação, circularidade dos dados, indução, dedução e abdução, utilização de memorandos, softwares e diagramas, presença de modelo teórico, e comparação com a literatura. O desenvolvimento das demais etapas compreendem na análise e discussão dos resultados, que consiste na seção seguinte.

\section{ANÁLISE E DISCUSSÃO DOS RESULTADOS}

Conforme já exposto, o presente estudo baseou-se em uma análise integrativa das publicações brasileiras na área de administração que utilizaram a TFD. Diante disso, esta seção busca apresentar e discutir a análise realizada nos artigos levantados, conforme já mencionado na metodologia do estudo. A Tabela 1 ilustra os principais achados em termos de caracterização da amostra analisada.
Observa-se que os artigos selecionados são oriundos em grande medida do Encontro Nacional da ANPAD (EnANPAD), evento considerado um dos mais relevantes da área da Administração no Brasil. Esse dado ressalta que os trabalhos desenvolvidos através da TFD têm sido debatidos em eventos científicos da área, e posteriormente publicados em periódicos, dentre os quais destacam-se a Revista Gestão.Org, a Revista de Administração Mackenzie e a Revista de Administração e Contabilidade da Unisinos. Ademais, verifica-se que os extratos das revistas se concentram entre A2 e B1, assim como observado por De Castro e Machado (2017). Esses resultados evidenciam a relevância e o impacto dos estudos que utilizaram o método para o seu campo.

Os resultados apontam também para uma redução nos últimos anos da utilização deste método na administração. Diante da amostra analisada, verifica-se que nos últimos quatro anos as publicações tem se mantido em dois artigos por ano. Semelhante aos achados deste estudo, Gomes et al. (2015) argumentam que na área da enfermagem a maioria dos artigos encontrados foram publicados a partir de 2008, sendo que se verifica apenas um estudo de 2004. Assim como Barreto, Garcia-Vivar e Marcon (2018) observaram uma concentração de $77 \%$ dos estudos no período entre 2012 e 2015.

Observa-se o mesmo fenômeno na administração, tendo em vista que os estudos foram publicados, em sua maioria, a partir de 2008, fato que corresponde com o ano de publicação da versão em português da obra de Strauss e Corbin (2008), aspectos verificados por Pinto, Freitas e Mendes (2012) e De Castro e Machado (2017). Porém, é possível observar estudos dos anos de 1980, 1990, 2004 e 2006. Sob um prisma internacional, Berto e Erdmann (2017) argumentam que grande parte dos estudos publicados em administração e que utilizaram o método, concentram-se entre 2009 e 2013, demonstrado também a influência da publicação da obra de Strauss e Corbin (2008). Ademais, é evidente o decréscimo da utilização da TFD nos últimos anos, que pode ser em parte justificada pelo tempo gasto com a elaboração da pesquisa e da sua publicação.

Destaca-se também que a maioria dos autores se concentram na região sul e sudeste, em centros como a USP, PUC-MG, FGV e UFSC. Em seu levan- 
tamento Gomes et al. (2015) levantaram que há maior é da região sul (67\%). Esses resultados evidenciam concentração em periódicos da região sudeste (58\%), a existência de grupos de estudiosos de TFD nessas enquanto que a origem dos autores, em grande parte, diferentes instituições de ensino.

Tabela 1 Identificação da amostra

\begin{tabular}{|c|c|c|c|c|c|c|c|c|c|}
\hline \multicolumn{2}{|c|}{ Revistas/Eventos } & \multicolumn{2}{|c|}{ Qualis } & \multicolumn{2}{|c|}{ Ano Publicação } & \multicolumn{2}{|c|}{ Origem autores } & \multicolumn{2}{|c|}{ Formação dos autores } \\
\hline EnANPAD & $6(24 \%)$ & $\mathrm{A} 1$ & $0(0 \%)$ & 2018 & $2(8 \%)$ & USP & $4(16 \%)$ & Doutores & $90(63 \%)$ \\
\hline GESTÃO.Org & $2(8 \%)$ & $\mathrm{A} 2$ & $6(35 \%)$ & 2017 & $2(8 \%)$ & PUC-MG & $3(12 \%)$ & $\begin{array}{l}\text { Doutoran- } \\
\text { do }\end{array}$ & $40(28 \%)$ \\
\hline $\begin{array}{l}\text { Revista de } \\
\text { Administração } \\
\text { Mackenzie }\end{array}$ & $2(8 \%)$ & $\mathrm{B} 1$ & $5(29 \%)$ & 2016 & $2(8 \%)$ & FGV & $3(12 \%)$ & Mestres & $4(3 \%)$ \\
\hline $\begin{array}{l}\text { BASE - Revista de } \\
\text { Administração e } \\
\text { Contabilidade da } \\
\text { UNISINOS }\end{array}$ & $2(8 \%)$ & B2 & $3(18 \%)$ & 2015 & $2(8 \%)$ & UFSC & $2(8 \%)$ & Graduados & $9(6 \%)$ \\
\hline $\begin{array}{l}\text { Revista de } \\
\text { Administração } \\
\text { Contemporânea }\end{array}$ & $1(4 \%)$ & B3 & $3(18 \%)$ & 2014 & $1(4 \%)$ & PUC-RIO & $1(4 \%)$ & Graduandos & $0(0 \%)$ \\
\hline $\begin{array}{l}\text { Revista Brasileira } \\
\text { de Marketing }\end{array}$ & $1(4 \%)$ & & & 2013 & $2(8 \%)$ & $\begin{array}{l}\text { Mackenzie - } \\
\text { Universidade } \\
\text { Presbiteriana } \\
\text { Mackenzie }\end{array}$ & $1(4 \%)$ & & \\
\hline $\begin{array}{l}\text { Revista Gestão \& } \\
\text { Planejamento }\end{array}$ & $1(4 \%)$ & & & 2011 & $4(16 \%)$ & USCS & $1(4 \%)$ & & \\
\hline $\begin{array}{l}\text { Revista de } \\
\text { Administração de } \\
\text { Empresas }\end{array}$ & $1(4 \%)$ & & & 2010 & $3(12 \%)$ & $\begin{array}{l}\text { Laureate } \\
\text { International } \\
\text { Universities }\end{array}$ & $1(4 \%)$ & & \\
\hline $\begin{array}{l}\text { Revista de Ciências } \\
\text { da Administração }\end{array}$ & $1(4 \%)$ & & & 2009 & $1(4 \%)$ & UNINOVE & $1(4 \%)$ & & \\
\hline $\begin{array}{l}\text { RACE: Revista de } \\
\text { Administração, } \\
\text { Contabilidade e } \\
\text { Economia }\end{array}$ & $1(4 \%)$ & & & 2008 & $4(16 \%)$ & UNICESUMAR & $1(4 \%)$ & & \\
\hline $\begin{array}{l}\text { Revista Ibero- } \\
\text { Americana de } \\
\text { Estratégia }\end{array}$ & $1(4 \%)$ & & & 2006 & $1(4 \%)$ & UFPE & $1(4 \%)$ & & \\
\hline $\begin{array}{l}\text { Brazilian } \\
\text { Administration Review }\end{array}$ & $1(4 \%)$ & & & 2004 & $1(4 \%)$ & UNIGRANRIO & $1(4 \%)$ & & \\
\hline EnEPQ & $1(4 \%)$ & & & & & FUCAPE & $1(4 \%)$ & & \\
\hline EnEO & $1(4 \%)$ & & & & & UFC & $1(4 \%)$ & & \\
\hline $\begin{array}{l}\text { Organizações \& } \\
\text { Sociedade }\end{array}$ & $1(4 \%)$ & & & & & IFRN & $1(4 \%)$ & & \\
\hline Cadernos EBAPE.BR & $1(4 \%)$ & & & & & UFU & $1(4 \%)$ & & \\
\hline $\begin{array}{l}\text { Revista de } \\
\text { Administração }\end{array}$ & $1(4 \%)$ & & & & & UNIVALI & $1(4 \%)$ & & \\
\hline
\end{tabular}

Fonte Elaboração própria. 
Além disso, observa-se que mais de $60 \%$ dos autores dos respectivos artigos, são doutores, e aproximadamente $30 \%$ são doutorandos, o que indica que esses autores estão ligados a Programas de Pós-Graduação, bem como demonstra a complexidade em relação a aplicação do método da TFD, haja vista que é, sobretudo, desenvolvida por pesquisadores mais experientes, o que fora observado também por Gomes et al. (2015).

Esses resultados demonstram que o método demanda muita dedicação e tempo do pesquisador, devido a imersão aos dados e a análise comparativa constante. Assim, poucos trabalhos dessa natureza são desenvolvidos em níveis de graduação ou mestrado, haja vista a necessidade de envolvimento dos pesquisadores e seu dispêndio de tempo (GOMES et al., 2015). Ademais, Roman, Osinski e Erdmann (2017) acrescentam que o processo de construção da teoria fundamentada é intenso e requer muito tempo do pesquisador, pois é necessário agendar entrevistas, analisar dados e redigir relatório, até que a saturação teórica seja atingida, processo que geralmente ultrapassa doze meses.

Diante desses resultados iniciais, constata-se que o método é pouco utilizado na área de administração, porém, quando utilizado, resulta em estudos com relevância em seu campo. Ademais, de certa forma, há uma concentração espacial, tanto nos periódicos em que são publicados, quanto na origem institucional dos autores. Os quais, em grande medida, são doutorandos ou doutores. Conforme já evidenciado, um dos aspectos que causam esse fenômeno pode residir na dificuldade e na demanda de tempo para o desenvolvimento dos estudos. Sobre isso, acredita-se que o método ainda é pouco explorado em eventos científicos da área, podendo ser trabalhado por meio de "workshops metodológicos", visando a introdução da TFD em estudos da administração. Ademais, para além da breve discussão acerca da TFD em disciplinas de metodologia em Programas de Pós-Graduação em Administração, evidencia-se a oportunidade de os programas ofertarem disciplinas específicas do método. A exemplo do Programa de Pós-Graduação em Enfermagem da Universidade Federal de Santa Catarina, que costuma ofertar uma disciplina de TFD para pesquisadores das mais diversas áreas, por demanda, acolhendo pesquisadores de outras instituições de ensino interessados em estudar o método.

Adicionalmente, a Tabela 2 evidencia as áreas de concentração dos estudos analisados. Sobretudo, verifica-se a concentração de pesquisas do campo de Estudos Organizacionais (28\% da amostra), os quais discutem temas sobre consciência regional, terceiro setor, governança, empreendedorismo, sustentabilidade, economia da comunhão e imagem organizacional.

Tabela 2 Áreas de concentração dos estudos

\begin{tabular}{l|c|c}
\multicolumn{1}{c|}{ Área } & Estudos & Percent. \\
\hline Estudos Organizacionais & 7 & $28 \%$ \\
\hline Finanças & 4 & $16 \%$ \\
\hline Gestão da Informação e conhecimento & 4 & $16 \%$ \\
\hline Marketing & 4 & $16 \%$ \\
\hline Gestão de Pessoas & 3 & $12 \%$ \\
\hline Estratégia & 2 & $8 \%$ \\
\hline Produção & 1 & $4 \%$ \\
\hline
\end{tabular}

Fonte elaboração própria.

Também, é possível observar estudos na área de Finanças (16\% da amostra), abrangendo temas como fusões e aquisições, gestão financeira e orçamento; Gestão da informação e conhecimento (16\%), englobando aspectos sobre ensino, evasão e transparência; Marketing (16\%), sobre temas como comportamento do consumidor e aprendizagem em marketing; Gestão de Pessoas (12\%), discutindo prazer e sofrimento no trabalho, competências e carreira; Estratégia (8\%), sobre adaptação e mudança organizacional; e, Produção (4\%), mais especificamente sobre sistemas de melhoria de desempenho operacional. Esses achados evidenciam que os métodos qualitativos de pesquisa estão sendo introduzidos em áreas tradicionalmente quantitativas, como finanças, marketing e produção. Demonstrando as possibilidades de análise dos mais diversos fenômenos organizacionais.

A partir da análise dos aspectos que caracterizam a amostra, parte-se para aspectos inerentes ao referencial metodológico dos estudos levantados, conforme a Tabela 3. 
Tabela 3 Informações acerca do referencial metodológico dos estudos

\begin{tabular}{l|c|c}
\hline & Sim & Não \\
\hline Vertente & $10(40 \%)$ & $15(60 \%)$ \\
\hline Referencial teórico & $18(72 \%)$ & $7(28 \%)$ \\
\hline Coleta de Dados & $25(100 \%)$ & $0(0 \%)$ \\
\hline Amostragem teórica & $14(56 \%)$ & $11(44 \%)$ \\
\hline Saturação dos dados & $13(52 \%)$ & $12(48 \%)$ \\
\hline Análise dos dados & $22(88 \%)$ & $3(12 \%)$ \\
\hline Codificação & $20(80 \%)$ & $5(20 \%)$ \\
\hline Circularidade dos dados & $9(36 \%)$ & $16(64 \%)$ \\
\hline Indução, dedução e abdução & $3(12 \%)$ & $22(88 \%)$ \\
\hline Memorandos & $6(24 \%)$ & $19(76 \%)$ \\
\hline Software de apoio & $16(64 \%)$ & $9(36 \%)$ \\
\hline Diagrama & $2(8 \%)$ & $23(92 \%)$ \\
\hline Modelo teórico/teoria & $17(68 \%)$ & $8(32 \%)$ \\
\hline Comparação com a literatura & $14(56 \%)$ & $11(44 \%)$ \\
\hline
\end{tabular}

Fonte Elaboração própria.

Primeiramente, em relação a vertente utilizada, observa-se que a maioria dos estudos (60\%) não especificaram a vertente utilizada. Dentre os estudos que descrevem a vertente utilizada, $80 \%$ seguiu a TFD Straussiana, e o restante (20\%) utilizou os fundamentos construtivistas do método com base em Charmaz (2006). O estudo de Pinto, Freitas e Mendes (2016) apontou que 70\% dos trabalhos não revelaram a sua vertente, por outro lado, aqueles que evidenciaram a vertente utilizada, a maioria seguiu a vertente Straussiana.

Diante da predominante utilização da vertente Straussiana na administração, e da concentração dos estudos a partir de 2008, explora-se a possibilidade de os autores terem escolhido a vertente não apenas pelas suas característica, pois esta é considerada mais estruturada operacionalmente, mas também pelo fato de que, naquele período, a referência mais atualizada em relação a TFD era da vertente Straussiana, em sua terceira versão (em inglês) no ano de 2007.

Considera-se a escolha da vertente como um elemento crucial para a aplicação do método, haja vista que apesar das principais vertentes apresentarem elementos em comum, as vertentes direcionam os pesquisadores sobre aspectos como a utilização da literatura, codificação e filosofia de pesquisa. Há de se considerar que os autores podem ter utilizado os elementos da TFD como técnica de coleta e análise de dados, mas não como o método como um todo.

Kenny e Fourie (2015) sustentam que o pesquisador não precisa necessariamente utilizar-se de uma forma pura da TFD, haja vista que dentro dos parâmetros de consistência, há uma liberdade entre os limites das três vertentes. Em um estudo desenvolvido pelos autores supracitados, inicialmente foi utilizada a vertente Straussiana, porém, na coleta e análise de dados, essa vertente mostrou-se muito rígida diante do tema estudado. Assim, os procedimentos de codificação foram afrouxados, aproximando-se da vertente Clássica, de forma que não violasse a integridade do método.

Dando sequência a análise, verifica-se que cerca de 70\% dos estudos analisados apresentam referencial teórico. Resultado que corrobora com o estudo de Castro e Machado (2017), que aponta que 75\% dos trabalhos analisados, apresentaram referencial teórico, construído antes da análise de dados. Esse resultado é coerente, haja vista que em grande parte utilizou-se as vertentes Straussiana e Construtivista do método. Conforme discutido anteriormente (Ver Figura 1), a identificação do problema de pesquisa na TFD Clássica é emergente e não necessita de uma revisão de literatura prévia. As demais correntes consideram uma aproximação com a literatura.

Em relação à coleta de dados, buscou-se verificar se essa etapa da pesquisa estava especificada no trabalho, o que foi observado na totalidade dos artigos, o mesmo resultado foi verificado por Gomes et al. (2015). Ademais, verificou-se que cerca de $56 \%$ dos estudos evidenciavam informações sobre a amostragem teórica, também observado em Gomes et al. (2015) e Barreto, Garcia-Vivar e Marcon (2018) na área da enfermagem. Ademais, comportamento semelhante foi observado a respeito da saturação teórica, ou seja, as coletas de dados foram realizadas até o momento de saturação teórica, corroborando aos achados de Barreto, Garcia-Vivar e Marcon (2018). Porém, Gomes et al. (2015) verificaram que 58,3\% dos estudos não discorreram acerca da saturação teórica.

$\mathrm{Na}$ sequência da coleta dos dados realiza-se a análise dos dados. Assim, dentre os estudos analisa- 
dos, a maioria detalha os processos utilizados (88\%). Esse achado vai ao encontro de Gomes et al. (2015) e De Castro e Machado (2017). Mas, em seu estudo na área de administração, Jacobus, De Souza e Bitencourt (2012) observaram na amostra analisada que a maioria dos estudos não demonstra igual empenho na descrição da coleta de dados, consequentemente, o mesmo ocorre em relação ao detalhamento da codificação dos dados.

Inerente a este ponto, ressalta-se a importância do delineamento das formas de codificação utilizadas, dessa maneira, $80 \%$ dos estudos evidenciam essas informações. Observou-se a evidenciação de variadas formas de codificação, como: aberta, axial e seletiva; aberta e axial; seletiva e aberta; inicial, focada e teórica; entre outras. Apesar disso, a maioria dos estudos segue os processos de codificação da TFD Straussiana (aberta, axial e seletiva).

Apesar da relevância da comparação constante ou circularidade dos dados na TFD, os estudos não evidenciam esse processo na sua redação (cerca de $65 \%)$. Esses resultados vão de encontro aos achados de Gomes et al. (2015) e de Barreto et al. (2018), que verificaram que na área de enfermagem a maioria dos artigos evidenciaram a utilização da análise comparativa constante. $\mathrm{O}$ mesmo foi observado nos demais levantamentos na área de administração, conforme evidenciado por Jacobus, De Souza e Bitencourt (2012) e De Castro e Machado (2017).

Cabe destacar que tanto a circularidade nos dados como a amostragem teórica consistem em elementos centrais da TFD, pois estes garantem que apenas os conceitos baseados nos dados direcionem-se a teoria, conforme argumentam Glaser e Strauss (1967). Além destas, a indução, dedução e abdução são outros fatores importantes na TFD, apesar que os estudos não costumam apresentar essas discussões em seu conteúdo (GOMES et al., 2015). Corroborando com esse achado, verifica-se que o mesmo ocorre na área de administração, pois aproximadamente $90 \%$ dos trabalhos não explicitam isso.

Outro aspecto relevante no desenvolvimento da TFD é construção e utilização de memorandos e diagramas. Destaca-se que na amostra analisada, apenas $24 \%$ indicaram a utilização de memorandos, e $8 \%$ a construção de diagramas pelo pesquisador. Resultado que vai ao encontro dos achados de Gomes et al. (2015), os quais argumentam que essa omissão das informações não significa que a construção e utilização não tenha ocorrido, pois é possível que o termo não tenha sido citado. Esses achados vão de encontro com o estudo de Barreto et al. (2018), o qual verificou-se que dentre os artigos analisados, $72,3 \%$ apresentam diagramas e $63,7 \%$ relataram a utilização de memorandos durante o processo de coleta e análise de dados.

Em se tratando de softwares utilizados nas pesquisas, destaca-se que aproximadamente $65 \%$ dos estudos evidenciaram a sua utilização, que em grande medida, consiste no software Atlas/ti e no NVivo, ambos voltados ao gerenciamento de dados qualitativos. Esses resultados vão de encontro aos achados de Gomes et al. (2015) e Pinto, Freitas e Mendes (2016). Os autores observaram que a maioria dos estudos analisados, $72 \%$ e $64 \%$ respectivamente, não evidenciaram ou não utilizaram software de apoio.

Considera-se que a utilização de diagramas, memorandos e de softwares de gerenciamento de dados qualitativos são de suma importância no processo de análise de dados. Devido a facilitação da organização dos dados e o seu processo de codificação. Quanto aos memorandos Glaser (1978) argumenta que eles são muito importantes, ao passo que os pesquisadores que não os utilizam, não estão fazendo TFD. Andrews et al. (2017) corroboram afirmando que os memorandos aumentam o nível conceitual e ajudam a integrar a teoria, e a elaboração dos diagramas da teoria são eficazes para a visualização de como os conceitos se relacionam entre si.

Um outro aspecto analisado foi a presença, ou não de um modelo teórico, destaca-se que buscou-se representações gráficas do modelo teórico. Porém suas categorias e relacionamentos poderiam estar expressos em texto apenas. E, mesmo que o método tenha como objetivo central a construção do modelo teórico, cerca de $32 \%$ dos estudos não o apresentaram. Enquanto que, em seu estudo Barreto et al. (2018) observaram o desenvolvimento de categoria central ou modelo teórico em aproximadamente $81 \%$ dos estudos. Gomes et al. (2015) verificaram que a metade dos estudos analisados apresentaram o modelo teórico. Adicionalmente, Berto e Erdmann (2017) verificaram em sua amostra que a totalidade dos artigos apresentam no final a teoria substantiva 
gerada em resposta aos questionamentos aos quais se propuseram.

Por um lado, a omissão do modelo teórico dificulta a validação da teoria, pois leva-se o modelo teórico construído - evidenciando as suas categorias e relações - até os profissionais da área, e ainda a um grupo de participantes da pesquisa (SANTOS et al., 2016). Por outro lado, conforme Bianchi e Ikeda (2008) destacam, a TFD não tem um compromisso, mas sim uma expectativa da busca por uma teoria fundamentada nos dados empíricos.

Por fim, na última fase da TFD tem-se a comparação da teoria emergente com a literatura. Diante desse aspecto, observa-se que apenas $56 \%$ dos estudos analisados efetivamente cumpriram com essa etapa do método. Corroborando com os achados em De Castro e Machado (2017), que observaram que 70\% dos estudos realizaram a comparação. Conforme já comentado, evidencia-se que as vertentes da TFD apresentam posturas distintas em relação com a literatura. A vertente Clássica, defende que o pesquisador deve suspender a consulta na literatura antes e durante a pesquisa. A consulta deve apenas ocorrer no momento da comparação da teoria no final do estudo. Por outro lado, Strauss e Corbin (1990) encorajam o uso da literatura, embora esta não fosse exaustiva, admitindo-se os seus benefícios (revelação de lacunas, fonte de dados, orientações e etc.). E, posteriormente, Charmaz (2006) aconselha adiar a elaboração da revisão de literatura até o instante em que o pesquisador começa a analisar os dados, para que a criatividade do pesquisador não fosse comprometida (KENNY; FOURIE, 2015).

\section{CONCLUSÃO}

O presente estudo objetivou apresentar uma síntese acerca do conhecimento que tem sido produzido na área de administração a partir da Teoria Fundamentada nos Dados, resultando um panorama acerca da utilização do método no país, identificando suas principais características, as quais possam ser utilizadas em estudos futuros acerca do método.

Diante dessa revisão integrativa é possível levantar diferentes informações advindas dos estudos realizados, como a definição do método e sua relevância no campo de estudo, áreas exploradas, bem como especificidades das diferentes vertentes, suas aproximações, e os elementos essenciais para o desenvolvimento deste. Conforme evidenciam Berto e Erdmann (2017) a TFD consiste em uma abordagem diferenciada e paradigmática que objetiva a compreensão de como os indivíduos se percebem de um contexto. Na Administração, utiliza-se a TFD para a geração de teoria substantiva, através de uma pesquisa interpretacionista.

Algumas evidências apontam para a utilização da TFD sem que suas etapas sejam cumpridas, ou evidenciadas. Apesar disso, o foco dos artigos analisados não foi o método em si, o que justifica o não aprofundamento sobre o método. Ademais, observou-se que os estudos têm sido discutidos em eventos científicos na área, bem como, publicados em revistas acadêmicas de relevância na área. Os resultados apontam também para uma redução nos últimos anos da utilização deste método na administração. Destaca-se também que a maioria dos autores se concentram na região sul e sudeste, e que na maioria são doutores. Esses resultados demonstram a complexidade do método, o qual demanda de muita dedicação e tempo do pesquisador, devido a imersão aos dados.

Apesar disso, os resultados possibilitaram um avanço no campo, pois a partir destes foi possível identificar a necessidade de intervenção no desenvolvimento do método na área de administração, pois fora observada utilizações parciais e a ausência de elementos essenciais para a implementação do método. Esses problemas observados seriam suprimidos através de uma maior discussão acerca do método na área, especialmente em eventos científicos e dentro dos programas de pós-graduação em administração, desmistificando e encorajando os pesquisadores a ingressar na TFD.

Em suma, evidencia-se a importância de estudos acerca da TFD, possibilitando o desenvolvimento do método nos mais diferentes campos de conhecimento, auxiliando os pesquisadores que venham a utilizar deste. Para estudos futuros sugere-se expandir a sua abrangência, ampliando os campos de conhecimento, e também abrangendo estudos realizados em outros países, buscando ter um diagnóstico mais completo da utilização da TFD. 


\section{REFERÊNCIAS}

ANDREWS, T.; MARIANO, G. J. D. S.; SANTOS, J. L. G. D.; KOERBER-TIMMONS, K.; SILVA, F. H. D. A metodologia da teoria fundamentada nos dados clássica: considerações sobre sua aplicação na pesquisa em enfermagem. Texto \& Contexto-Enfermagem, v. 26, n. $4,2017$.

BANDEIRA-DE-MELLO, R.; CUNHA, C. J. C. A. Grounded Theory. In: GODOI, C. K.; BANDEIRADE-MELLO, R.; SILVA, A. B. (Organizadores). Pesquisa Qualitativa em Estudos Organizacionais: Paradigmas, Estratégias e Métodos. São Paulo: Editora Saraiva, 2006.

BARRETO, M. da S.; GARCIA-VIVAR, C.; MARCON, S. S. Methodological quality of grounded theory research with families living with chronic illness. International Journal of Africa Nursing Sciences, v. 8,2018 .

BERTO, A. M.; ERDMANN, R. H. Grounded Theory gerando teorias na Administração. In: Simpósio de Administração da Produção, Logística e Operações Internacionais, 2017, São Paulo. Anais do XX SIMPOI. São Paulo: FGV EAESP, 2017.

BIANCHI, E. M. P. G.; IKEDA, A. A. Usos e aplicações da grounded theory em administração. GESTÃO. Org-Revista Eletrônica de Gestão Organizacional, v. 6, n. 2, 2008.

CHARMAZ, K. Constructing grounded theory: A practical guide through qualitative analysis. London, UK: Sage Publications, 2006.

CHIZZOTTI, A. A pesquisa qualitativa em ciências humanas e sociais: evolução e desafios. Revista Portuguesa de Educação, 2003, 16. Disponível em: http://www.redalyc.org/articulo.oa?id=37416210. Acesso em: 02 out. 2018.
DE CASTRO, A. R.; MACHADO, L. GROUNDED THEORY: UMA ANÁLISE DA PRODUÇÃO CIENTÍFICA BRASILEIRA EM ADMINISTRAÇÃO NO PERÍODO DE 2000 A 2014. Revista Alcance (Online), v. 24, n. 2, p. 258, 2017.

GLASER, B. G. Theoretical Sensitivity: Advances in the Methodology of Grounded Theory. Califórnia: Mill Valley, 1978.

GLASER, B.; STRAUSS, A. The discovery of grounded theory: strategies for qualitative research. New York: Aldine Transaction, 1967.

GOMES, I. M.; HERMANN, A. P.; WOLFF, L. D. G.; PERES, A. M.; LACERDA, M. R. Teoria fundamentada nos dados na enfermagem: revisão integrativa. Revista de Enfermagem UFPE on line, v. 9, n. supl 1, p. 466-74, 2015.

HALAWEH, M. Using grounded theory as a method for system requirements analysis. Journal of Information Systems and Technology Management, v. 9, n. 1, p. 23-38, 2012.

HARRIS, T. Grounded theory. Nursing Standard, v. 29, n. 35, p. 32-39. 2015.

HEATH, H.; COWLEY, S. Developing a grounded theory approach: a comparison of Glaser and Strauss. International journal of nursing studies, v. 41, n. 2, p. 141-150, 2004.

HUNTER, A.; MURPHY, K.; GREALISH, A.; CASEY, D.; KEADY, J. Navigating the grounded theory terrain. Part 1. Nurse researcher, v. 18, n. 4, 2011.

IKEDA, A. A.; BIANCHI, E. M. P. G. Considerações sobre usos e aplicações da grounded theory em administração. Revista de Administração FACES Journal, v. 8, n. 2, 2009.

KENNY, M.; FOURIE, R. Contrasting classic, Straussian, and constructivist Grounded Theory: Methodological and philosophical conflicts. The Qualitative Report, v. 20, n. 8, p. 1270-1289, 2015. 
LOURENÇO, D. D. S.; FERREIRA, C. A.; ROSA, A. R. ETNOGRAFIA E GROUNDED THEORY NA PESQUISA DE MARKETING DE RELACIONAMENTO NO MERCADO CONSUMIDOR: UMA PROPOSTA METODOLÓGICA. RAM. Revista de Administração Mackenzie, v. 9, n. 4, 2008.

MELLO, R. B.; CUNHA, C.J.C.A. Grounded theory. In.: GODOI, C. K.; MELLO, R. B.; SILVA, A. B. (Orgs). Pesquisa qualitativa em estudos organizacionais: paradigmas, estratégias e métodos. $2^{\mathrm{a}}$ ed. São Paulo: Saraiva; 2010.

MENDES, K. D. S.; SILVEIRA, R. C. D. C. P.; GALVÃO, C. M. Revisão integrativa: método de pesquisa para a incorporação de evidências na saúde e na enfermagem. Texto \& Contexto-Enfermagem, v. 17, n. 4 , p. 758-764, 2008.

MENDONÇA, A. T. B. B.; REMONATO, R. L. C.; MACIEL, C. O.; BALBINOT, Z. Grounded Theory. In: TAKAHASHI, A. R. W. (Org.) Pesquisa Qualitativa em Administração: Fundamentos, métodos e usos no Brasil. São Paulo: Atlas, 2013.

MIGUEL, L. A. P.; POPADIUK, S. Integrando metodologias na análise de dados sob o paradigma interacionista simbólico: um caso prático. Cadernos EBAPE. BR, v. 12, n. 2, p. 357-373, 2014.

PETRINI, M.; POZZEBON, M. Usando Grounded Theory na construção de modelos teóricos. Gestão \& Planejamento, v. 10, n. 1, 2009.

PINTO, M. de R.; FREITAS, R. C. de; MENDES, C. A. F. Grounded theory in management studies in Brazil: among the plurality of strands, improper uses and mistaken understanding?. Revista Gestão \& Tecnologia, v. 16, n. 1, p. 33-54, 2016.

PINTO, M. de R.; SANTOS, L. L. da S. A grounded theory como abordagem metodológica: relatos de uma experiência de campo. Organizações \& Sociedade, v. 19, n. 62, 2012.
PINTO, M. de R.; SANTOS, L. L. da S. Em busca de uma trilha interpretativista para a pesquisa do consumidor: uma proposta baseada na fenomenologia, na etnografia e na grounded theory. RAE-eletrônica, v. 7, n. 2, 2008.

PREDEBON, E. A.; DE SOUSA, P. D. B. The feasibility of grounded theory methodology on Brazilian public administration research. Revista de Administração, v. 4, n. 3, p. 16-30, 2006.

PREDEBON, E. A.; RITOSSA, C. M.; DE SOUSA, P. D. B.; VERDU, F. C.; AGUIAR, E. C. GROUNDED THEORY: MELHORANDO A PRATICA E A PESQUISA EM ADMINISTRAÇÃO NO BRASIL. Revista de Administração FACES Journal, v. 10, n. 3, 2011.

ROMAN, D. J.; OSINSKI, M.; ERDMANN, R. H. The construction process of grounded theory in administration. Contaduría y Administración, v. 62, n. 3, p. $985-1000,2017$.

SANTOS, J. L. G. Novas possibilidades da Teoria Fundamentada nos Dados para a pesquisa em enfermagem. Revista de Enfermagem da UFSM, v. 8, n. 2, p. 206-208, 2018.

SANTOS, J. L. G. D.; ERDMANN, A. L.; SOUSA, F. G. M. D.; LANZONI, G. M. D. M.; MELO, A. L. S. F. D.; LEITE, J. L. Perspectivas metodológicas para o uso da teoria fundamentada nos dados na pesquisa em enfermagem e saúde. Escola Anna Nery, v. 20, n. 3, 2016.

STRAUSS, A.; CORBIN, J. M. Basics of qualitative research: Grounded theory procedures and techniques. Sage Publications, Inc, 1990.

STRAUSS, A.; CORBIN, J. Basics of qualitative research: Techniques and procedures for developing grounded theory (2nd ed.). Thousand Oaks, CA: SAGE publications, 1998. 
Angélica Pott de Medeiros • José Luís Guedes dos Santos • Rolf Hermann Erdmann

STRAUSS, A.; CORBIN, J. Pesquisa qualitativa: técnicas e procedimentos para o desenvolvimento de teoria fundamentada. Tradução Luciane de Oliveira da Rocha. 2 ed. - Porto Alegre: Artmed, 2008. 